\title{
Hedging your bets in a volatile world: an introduction to the use and pricing of European call options.
}

\section{Gearóid Ryan}

School of Mathematical Sciences, UCC

\section{Introduction}

In recent years, our economy and livelihoods have been affected by the volatility of financial markets. We have seen how banks and investors have failed to control and understand their risks. In this article, I outline one of the fundamental tools used to control exposure to risk in financial markets. In doing so, I explore some of the underlying assumptions of mathematical models used in the financial industry today and show how my research attempts to make these assumptions more realistic while keeping the model simple.

As well as the trading of stocks and shares, many of the transactions in financial markets consist of the trading of contracts. Such a contract may be an agreement between two parties on the price of a stock, the quantity and time of delivery of some commodity, or contracts that provide insurance for investors. My research is concerned with determining the value of one of the simplest kinds of financial contracts. The name of the contract is a 'European Call Option'. This is, very simply, a contract between two parties to trade some product in the future. The date of the transaction and the price of the product are agreed in the contract. It is important to note that the buyer of the product, i.e., the person who owns the contract, has no obligation to go ahead with the transaction if he feels he can do better elsewhere. In other words, with this contract, he has the option of buying the product for the specified price, at the specified time.

We study this simple contract because understanding it will pave the way towards an understanding of more complicated contracts. We will see how a European Call Option can be used to hedge exposure to volatility, and how to assign a value to this contract.

\section{Background information}

Before discussing financial contracts, it is necessary to explain how financial mathematicians understand stock prices. The most basic mathematical models for stock prices assume that the price grows naturally over time but is subject to random fluctuations. The 
technical name for this is Geometric Brownian Motion. This model was first introduced in 1900 by Louis Bachelier, a French mathematician.

For example, imagine we have a bank account whose value at the end of the year is $€ 100$. Our friendly bank manager adds $€ 5$ to our account as interest. During the year our bank balance has fluctuated due to random bills and lodgements, the size of which can't be predicted beforehand. The price of a stock, according to Bachelier's model, behaves in a similar way to our bank balance; it has a predetermined growth rate (the interest added), and is subject to random fluctuations. The model goes slightly further in that it assumes, using the above analogy, that the chances of having of a large change in our bank balance is much more unlikely than having a smaller one. You may think of a small change as day to day purchases on your laser card such as buying a cup of coffee, paying for petrol etc., and a large change as the bimonthly electricity bill.

The word volatility refers to the size of the random fluctuations of the stock price. A highly volatile stock will have large fluctuations in its price, making the future price more unpredictable. In a mathematical sense, one measure of the volatility could be the variance of the stock price. The biggest assumption in Bachelier's model is that the volatility of the stock price is constant. We can think of this in terms of coin tossing. In a coin toss the chances of getting tails is always $50 \%$. This is obvious, but the mechanics of financial markets is much more complicated than a simple coin toss and it has been shown that the chances of certain events happening, such as the price of a stock going up or down, changes regularly. The market is like a coin, whose chances of getting tails changes every time it is tossed, and may even depend on previous tosses.

\section{My research}

As mentioned above, the goal of my research is to find the value of European Call Options. The most important aspect of this research is having a very good stock price model. After that, finding the value of simple financial contracts is relatively straightforward.

One large area of current research in mathematical finance is in relaxing the assumption of constant volatility in stock price models, and this is the area in which I work. We can see from Figure 1 that this assumption is unrealistic. Building on previous research I assume that the stock obeys the same equations as Geometric Brownian Motion except that the parameter in my model that represents the volatility is constantly changing. In fact, I assume that the volatility of the stock price also behaves according to Geometric Brownian Motion. This is called a 'stochastic volatility model'.

Figure 1 Price in USD of AIB shares from 2003 to 2008. We see that the size of the fluctuations in the share price is increasing with time i.e., the volatility is increasing. This data suggests that stock price models should not assume constant volatility. ( ${ }^{1}$ Source: 
Yahoo Finance)The next important point to note is that I assume the underlying source of randomness in both the stock price and the stock price's volatility is the same. For example, changes in the price of petrol and the price of diesel are for the most part subject to changes in the price of oil, i.e., they are subject to the same underlying source of randomness.

My model also takes into account historic information. It assumes that the volatility in stock price depends on previous price fluctuations and previous volatility. This information comes from existing market data. The model puts more importance on more recent data, as it is obvious for instance that the stock price yesterday is far more relevant than the price one year ago.

My research is on improving existing models by looking at the historic information in new ways and by looking at how to best weigh the importance of this information.

With this new model, I hope to provide better predictions of the values of financial contracts. This research is both numerical and theoretical. The numerical part is where computer algorithms are used to refine the parameters of our model. The algorithms use existing market data to do this. The theoretical part is in the designing of the model and in devising methods to calibrate its parameters.

\section{Hedging your risk}

As an example of how European Call Options are used we imagine a manufacturer who, in planning for the year ahead knows he can afford to buy some raw material for, at the most, $€ 100$ in 6 months time. If the price is higher than this the manufacturer will go broke. The manufacturer knows that the price of the raw material is subject to large fluctuations/high volatility and needs to find a way of protecting himself against this.

The manufacturer may buy a European Call Option contract from one supplier that guarantees them a price of $€ 100$ for the raw material in 6 months time. The manufacturer pays the supplier a small fee for this contract. Now if in 6 months time, the price of the raw material on the market is greater than $€ 100$, the manufacturer will use the contract and buy the material from the supplier for $€ 100$. If the value of the raw material is less than $€ 100$, then the Call Option is worthless as the raw material can be bought from the market at the lower price. In this way, the contract has allowed the manufacturer to hedge against future volatility and eliminates a source of risk.

Now imagine that we are the supplier. As the value of the raw material stored in our warehouse goes up, the value of our company goes up. The raw material is an asset. On the other hand, as the value of our raw material goes up, the more likely the contract we have written will be used, forcing us to sell for a lower than market price. The contract is a liability. 
Of course, the changes in price of our raw material and the change in value of these contracts are linked. In fact, one can determine the best ratio of raw material and contracts to own such that the price movements of each perfectly cancel out each other. No matter what the change in price of the raw material, the net value of the suppliers company will always stay the same. This perfect ratio needs to be recalculated as frequently as possible.

The big question here is what fee should the supplier charge the manufacturer, i.e., what is the value of the contract. Finding a fair value is the goal of my research.

\section{Conclusions}

This research area aims to model the uncertainty of financial markets and in doing so provide tools that help manage or eliminate sources of risk. The work on pricing the European Call Option is a starting point to understanding more complicated contracts. The tools developed here may be useful to many businesses that are exposed to uncertainty. The current economic situation highlights the importance of this area of research and indicates how much we have yet to learn.

I would like to thank my supervisor Dr. Tom Carroll and Dr. Gregory Temnov for helping me in the preparation of this article and to acknowledge the Edgeworth Center for Financial Mathematics and Science Foundation Ireland for funding my research. 